\title{
PSYCHISCHE WIRKUNGEN CHEMISCHER SUBSTANZEN UND IHRE PSYCHIATRISCHEN ANWENDUNGEN*
}

\author{
Von \\ W. Mayer-Gross
}

Aus der Abteilung für experimentelle Psychiatrie, Medical School, University of Birmingham, England

\section{Historisches}

Bis in die jüngste Zeit waren in der Pharmakologie psychische Wirkungen chemischer Substanzen unerwünscht oder wurden geflissentlich übersehen. Sie komplizierten und störten die Beobachtung z. B. von Drogen mit Effekt am vegetativen Nervensystem und erschwerten die Deutung körperlicher Phänomene. Darin ist nun plötzlich eine Wandlung eingetreten; wie kam es dazu und was hat dieses Interesse zu bedeuten?

Als Zweige einer wissenschaftlichen Medizin mit eigenen Problemen und Methoden sind Pharmakologie und Psychiatrie relativ jung. Systematische Forschung auf beiden Gebieten begann etwa am Ende des 18. und in der ersten Hälfte des 19. Jahrhunderts; aber während Pharmaka als ein wesentlicher Tei medizinischer Praxis fast so alt sind wie die Heilkunst selbst, blieb die Seele und ihre Störungen im wesentlichen außerhalb der Medizin in den Händen der Magier, Priester und schließlich der Philosophen; noch KANT beanspruchte die Beurteilung geistiger Abnormalitäten als ihr Feld.

Dies erklärt zum Teil. warum Chemikalien mit vorwiegend psychischer Wirkung, ,Psychoto-Mimetica", außerhalb der Medizin verblieben, obwohl sie in allen Teilen der Welt seit uralten Zeiten ein Bestandteil des täglichen Lebens bildeten. $\mathrm{Zu}$ den Substanzen gehören der Alkohol in all seinen Formen, Kaffee, Tee, Opium und seine Verwandten, Haschisch, Coca und zahlreiche seltenere Drogen. Die Mehrzahl sind Stimulantien, die körperliche und geistige Tätigkeit erleichtern durch die Erzeugung guter Stimmung, Anregung der Phantasie und eines Kraftgefühls. Sie waren PLrnius" ,Mittel wider die Unbeständigkeit des Gernüts", und ihre Herkunft wurde mythisiert (BERINGER 1948).

In magisehen und religiösen Zeremonien wurden ihre Wirkungen ausgenutzt: sie halfen, die Götter zu zitieren; sie erzeugten getrübte oder veränderte Bewußtseinszustände und vermittelten die Verbindung mit übernatürlichen Kräften; damit rückten ihre Wirkungen außerhalb der nüchternen und unpersönlichen Sphäre der Wissenschaft. Noch heutzutage ist das Trinken von Alkohol in manchen Kreisen die Initiationszeremonie der Männlichkeit, und die Diskussion der Alkoholwirkung bringt die Beteiligten aus dem Gleichgewicht. Ärzten fehit oft ein ruhiges Urteil, wenn es sich um die Trinkgewohnheiten ihrer Patienten handelt; sie sind entweder blind und tolerant oder dogmatisch und streng.

* Vortrag auf der 100. Versammlung der Gesellschaft Deutscher Naturforscher und Ärzte, 2. Oktober 1958 in Wiesbaden.

Klin. Wsohr., 37. Jahrg.
Hinzu kommt die soziale Funktion vieler Psychomimetica, sie fördern die frohe, gesellige Haltung und helfen, wenigstens für ein paar Stunden, die sozialen Schranken zwischen Menschen und Klassen zu überwinden. Sie erleichtern die Bürde des Daseins am Tagesende. Andere, wie Opium und Haschisch, helfen uns, den Forderungen und Sorgen der Wirklichkeit auszuweichen und ins Reich der Wünsche und Träume zu entfliehen.

Dem wissenschaftlich interessierten Pharmakologen war der stimulierende Effekt auf die Psyche relativ uninteressant; er konzentrierte sich auf die beruhigende, einschläfernde und narkotisierende Wirkung von Substanzen, die für Schmerzlinderung und Anaesthesie in der Chirurgie von praktischer Wichtigkeit waren.

Die Psychologie der Rauschmittel blieb anekdotisch und unsystematisch, bis Krampelin 1892 seine Experimente begann mit dem Rüstzeug der jungen, von WUNDT begründeten, experimentellen Psychologie. Ihre Ergebnisse weckten die Erwartung, da $\beta$ das Seelische mit den anderen Lebenserscheinungen bald ein Studienobjekt im neuen naturwissenschaftlichen Weltbild sein werde. Krafepelin untersuchte die Wirkung von Alkohol, Morphium, Coffein und anderen Drogen auf seelische und körperliche Arbeitsleistung. Aber er veranlaßte auch Experimente mit psychoseerzeugenden Drogen, wie z. B. Meskalin, das LEWIN entdeckt hatte und zur Gruppe der Phantastica zählte. Kraepelins Schüler, Wimmanns und Beringer (1927), unternahmen in den 20er Jahren in Heidelberg Serien-Experimente mit Meskalin an normalen Versuchspersonen, deren eingehende Schilderungen in unübertroffener und musterhafter Weise die psychologische Wirkung der Droge analysierten; das medizinische Ergebnis dieser Versuche war gering. Nur mit Vorbehalt konnte man sagen, daß sie ein besseres Verständnis der psychiatrischen Patienten ermöglichten.

Eine ätiologische Aufklärung oder therapeutische Anwendung war wohl ein Wunschbild der Versuche, in weiter Ferne gesehen. Gewisse Ähnlichkeiten mit Episoden in der beginnenden Schizophrenie wurden erörtert; aber die Idee einer vergleichenden Störung der chemischen Vorgänge im Gehirn ging nicht viel weiter als der Begriff der ,Stoffwechselstörung“" unter dem Kr.EPELIN in einer frühen Auflage seines Lehrbuches die endogenen Psychosen eingeordnet hatte.

Von den Experimenten mit Meskalin, Hasschisch, Hysoscin und Stickoxydul abgesehen, die deutsche Psychiater in den 20iger Jahren anstellten, ging wenig Anregung in das pharmakologische Feld. Hier 
beschäftigte man sich einzig und allein mit der Ausschaltung und Unterdrückung psychischer Symptome. Die Pharmakologie versah die Psychiatrie mit Beruhigungs- und Schlafmitteln, mit der „,chemischen Zwangsjacke" und dem Dauerschlaf. Erst 1935 erschien die erste, zentral stimulierende Substanz in den Pharmalkopöen der Welt in Gestalt des Amphetetaminsulphats, das charakteristischerweise schon über 20 Jahre lang als ein Stimulans bekannt gewesen war. Zuerst gegen die Schlafanfälle der Narkoleptiker angewandt, wurde seine weckende und anregende Eigenschaft bald erkannt und machte es populär. Als Mittel gegen Erschöpfung im Krieg, gegen Examensangst und Lampenfieber war auch seine Anwendung zeitweise außerhalb des ärztlichen Bereiches. Wie sehr Umgebung und soziale Einflüsse die Verbreitung und Wirkung psychisch wirksamer Mittel beeinflussen, zeigt das Beispiel Japans. Dort ist die Sucht der ,Weckamine“ ein weit verbreitetes soziales Utbel und als solches von den Behörden bekämpft.

Eine Reihe verschiedener Faktoren hat im Laufe des letzten Jahrzehnts zu dem Aufbliuhen des Interesses an psychologischen Fragen geführt, das auch auf die Arzneimittelkunde übersprang. Die erfolgreiche Bekämpfung der Infektionskrankheiten und ihrer Gefahren haben die Rolle seelischer Störungen in der Medizin klarer hervortreten lassen. Die verlängerte Lebensdauer vermehrte die Zahl psychiatrischer Patienten jeder Art, nicht nur der altersspezifischen, senilen und arteriosklerotischen Kranken. Die Aussiebung seelisch Labiler und Unterdurchschnittlicher in den Armeen des 2. Weltkrieges zeigte ihren erheblichen Anteil an den Halb- und GanzInvaliden in allen Ländern. Die Bettenzahl für psychiatrische Fälle, über $40 \%$ des Gesamts, zeigte die Belastung des sozialen Dienstes durch psychiatrische Erkrankungen in einem „welfare state" wie Großbritannien.

Inzwischen hat die Neurophysiologie, wie JUNGS Referat zeigte, vieles über die Tätigkeit des peripheren und zentralen Nervensystems aufgeklärt. Der Stoffwechsel des Gehirns und der Nervenzelle wurde durch Fortschritte der Biochemie besser verstanden, dank der Entdeckungen von EMDen, KReBs und anderer auf dem Gebiete der Kohlenhydrat-Verwertung.

Hier scheint es angebracht, an JoHanN LUDwIG Thunrснuм, den Pfarrerssohn aus Büdingen, zu erinnern, der in Gießen doktorierte und ein Schüler LIEBIGs war. Fr verfaßte 1884 das erste und auf lange Zeit hinaus das einzige Buch über die chemische Konstitution des Gehirns. Nachdem er 1853 aus politischen Gründen nach London ausgewandert war, entfaltete er dort eine erfolgreiche Tätigkeit als Arzt und Wissenschaftler; seine Arbeiten waren vorwiegend um die Anwendung der Chemie in der Medizin besorgt. Er starb 1901, beglückt von der Übersetzung seines Hauptwerkes ins Deutsche, die noch vor seinem Tode erschien (DrabKIN 1958).

\section{Neue Pharmaka und Theorien}

Ausschlaggebend für das pharmakologische Interesse in der Psychiatrie war aber wohl die Auffindung neuer Mittel mit unerwarteter psychischer Nebenwirkung; zufällig, wie im Falle des Lysergsäure-Ab- kömmlings LSD 25, oder auf der Suche nach wirksameren Narkotica, wie im Falle der PhenothiazinDerivate. Die fundamentale Bedeutung der Entdeckung von A. Hofmann (1943) wurde auch von den Psychiatern nur langsam erfaßt; es war ein Amid der natürlichen Lysergsäure, zum Teil synthetisch erzeugt (STOLL 1947). In der Menge von ${ }^{1 / 100} \mathrm{mg}$ ruft es einen psychoseartigen Zustand hervor, der in vielem der Modellpsychose mit Meskalin gleicht, wovon aber $0,5 \mathrm{~g}$ injiziert oder geschluckt werden müssen, um eine ähnliche Wirkung hervorzurufen. Verglichen mit LSD 25 ist Meskalin eine chemisch einfache Substanz, verwandt mit Stoffen wie Tyrosin und Adrenalin, die sich normalerweise im Körper finden.

Die Phenothiazin-Gruppe war wegen ihrer Antihistamin-Wirkung für den Pharmakologen von Interesse; es war die schläfrigmachende Nebenwirkung, die das Interesse von LABonIT und anderen französischen Forschern anzog und zur Verwendung in Narkosen mit Unterkühlung der Körpertemperatur führte. Ein „Winterschlaf-Cocktail“" war das Ergebnis dieser Versuche, die DrLay in Paris auf das psychiatrische Gebiet anwandte. Nach mancherlei Enttäuschungen stellte sich heraus, daß hier ein neues Beruhigungsmittel gefunden war, das gerade auf die chronischen Psychosen wirkt, die wegen ihrer Erregung und Gewalttätigkeit über Jahre in den Nervenkrankenhäusern zurückgehalten werden müssen und dort die Pflege und die ärztlichen Aufgaben so sehr erschweren. Zu einer Zeit, wo die Überfüllung der Anstalten und der Mangel an geeignetem Pflegepersonal ein welt. weites soziales Problem aufwarf, bot uns die Pharmakologie eine zwar nicht ätiologische, aber praktische, symptomatische Lösung an. Die "neuro-leptische“" Droge Chlorpromazine (Megaphen) steht hinter den Reformern, die die geschlossenen Abteilungen der Anstalten öffnen und die Familienpflege anstelle der langen Verwahrung hinter Krankenhausmauern anstreben. Neben Megaphen müssen hier die Rauwolffia-Präparate genannt werden, Abkömmlinge einer uralten Droge aus Indien; das Reserpin, das in jüngster Zeit aus der Droge isoliert und in seiner chemischen Natur bekannt wurde, hat offenbar eine ähnliche beruhigende Wirkung auf die Symptome der chronisch erregten Patienten - und verkürzt in vielen Fällen auch die akute Psychose.

Wie diese psychologische Wirkung zustande kommt, was für ein Hirnmechanismus die eigenartige Beruhigung und seelische Kühle mit Distanzierung von den krankhaften Ideen erzeugt, die den Kranken vorher erfüllt und beherrscht haben, ist heute noch unbekannt.

Ein Patient schrieb Dutzende von beschimpfenden Briefen an den Direktor der Anstalt, in denen er fast täglich sich über die ungesetzliche Zurückhaltung eines völlig gesunden Menschen im Hospital bitter beschwerte. Nach etwa 3 Monaten Behandlung mit „Serpasil" änderte sich von einem Tag auf den anderen der Ton seines Briefes: or sehe doch jetzt ein, daß er krank gewesen sei und sich manches eingebildet habe und daß vieles, was man für ihn getan habe, zu seinem Besten geschehen sei; er bat um Entschuldigung und um weitere Behandlung als freiwilliger Patient. Allmählich schwanden die Symptome der Erkrankung, Halluzinationen und Wahn ideen traten in den Hintergrund, und der Kranke, der seit 7 Jahren an einer paranoiden Schizophrenie gelitten hatte, konnte, frei von Symptomen, zu seiner Familie entlassen werden. Die Remission hat bis in die letzte Zeit, d. h. jetzt 5 Jahre, angehalten. 
Noch gibt es viele Fälle, die nicht so prompt und erfolgreich reagieren - und wie MANFred BLEUler (1958) jüngst wieder betonte, von einer spezifischen und ätiologischen Behandlung der Schizophrenie sind wir noch weit entfernt. Es ist aber zu bedenken, daß symptomatische Behandlung psychologischer Störungen oft von viel größerer Bedeutung für den Kranken ist als die symptomatische Behandlung in der inneren Medizin, weil das Symptom in vielen Fällen das Leiden beherrscht und damit das Leben des Patienten bestimmt. Symptomatische Behandlung der Erregbarkeit des Blutdruckkranken kann lebensrettend sein oder doch auf lange Zeit sein Schicksal bestimmen.

Megaphen und Reserpin haben außer ihrem distanzierenden und beruhigenden EinfluB auf die Psychose wichtige Nebenwirkungen im Zentralnervensystem von besonderem theoretischem Interesse: Beide erzeugen in größeren Dosen vorübergehende extrapyramidale Symptome in Gestalt der Paralysis agitans. Reserpin kann außerdem in seltenen Fällen nach längeren Gaben das Bild einer Depression mit Hemmung und tiefer Verstimmung auslösen. Diese Nebenwirkungen zeigen, daß es sich hier um Mittel handelt, die an anderen Systemen des Zentralorgans angreifen als die bekannten Sedativa und Hypnotica. Daß sie nicht nur eine auslöschende, negative Wirkung auf Symptome haben, sondern auch solche erzeugen, rückt sie in die Nähe der Substanzen, mit deren Hilfe wir die Modellpsychosen hervorrufen, von denen man seit langem Auflzlärung über die natürlichen Geistesstörungen erwartet. -

Drei theoretische Ansätze aus den letzten Jahren mögen illustrieren, wie biochemische Ideen zur Hypothesenbildung auf unserem Gebiet führen und wie weit sie fruchtbar waren. Unter Hinweis auf die chemische Verwandtschaft zwischen Meskalin und Adrenalin vermutete eine Gruppe von Forschern in Kanada, unter der Leitung von Hofrer (1954), daß eine in dem Patienten erblich angelegte Stoffwechselanomalie zu einem Abbauprodukt des Adrenalin führen könnte, das die schizophrene Krankheit erzeugte. Angeborene Stoffwechselanomalien mit Einfluß auf die Funktion des Nervensystems sind bekannt, z. B. die Phenylketinuria mit Schwachsinn. Adrenochrom ist ein Zwischenprodukt des Adrenalin. stoffwechsels, und HoFFER und seine Mitarbeiter versuchten, seine psychoto-mimetische Aktion nach. zuweisen - bis dato nicht mit vollem Erfolg. Aber auch, wenn solch ein Zwischenprodukt im Blutserum nicht nachzuweisen ist, bestünde ja die Möglichkeit, daß es im Gehirn selbst gebildet wird und dort $a b$ norme Bedingungen für die psychische Verwertung der Unwelt erzeugt, ehe es völlig abgebaut und ausgeschieden wird.

Ein zweiter theoretischer Ansatz, ebenfalls bis dato nicht beweiskräftig, gruppiert sich um das neue Hormon Serotonin, wovon JUNG berichtet hat. Es ist chemisch mit Reserpin verwandt, und gröBere Gaben der Droge lassen ein Spaltungsprodukt des Serotonin im Urin des Patienten erscheinen. Ein Zusammenhang zwischen Reserpin-Wirkung und Serotonin-Abbau schien plausibel. Darüber hinaus zeigte sich in Tier. präparaten ein Antagonismus zwischen Serotonin und LSD 25, so daß eine Zeitlang die psychoseerzeugende Wirkung von LSD 25 über das Serotonin erklärbar erschien; bis sich herausstellte, daß gewisse Lysergsäure-Derivate ohne psychotische Wirkung viel stärkere Antagonisten des Serotonin im biologischen Präparat sind. Trotzdem kann vom Studium des Serotonin-Stoffwechsels weitere Aufhellung der psychologischen Arzneiwirkung erwartet werden.

Die Undurchlässigkeit der Blut-Hirnschranke für größere Moleküle wurde ebenfalls von JUNG erwähnt and in ihrer Bedeutung beschrieben. CARL Pffeiffer (1957) von der Emory University in Georgia fußt eine Arbeitshypothese auf der Annahme, daß tertiäre Amine die Schranke passieren, während quarternäre zurückgehalten werden und daher keine zentrale Wirkung haben können. Er suchte nach einer Substanz, die wie Cholin bei dem zentralen Aufbau von Acetylcholin hilft, dessen Rolle als zentrales Stimulans er für erwiesen hält, und zugleich die Schranke leichter als Cholin passiert. Hier handelt es sich nicht um ein schnell wirkendes Medikament, sondern um ein Anregungsmittel, das PfeIfFer zunächst einmal in einem 6wöchigen Experiment mit Studenten sorgfältig ausprobierte, mit Leertabletten und allen Vorsichtsmaßregeln gegen Suggestion und Autosuggestion von Versuchsleitern und Versuchspersonen. Der Einfluß auf Muskeltonus, geistige Arbeit und Schlaf war eindeutig. Dann ging er dazu über, die Substanz bei schizophrenen Patienten zu prüfen - bis dato mit nicht viel mehr als ermutigendem Ergebnis.

Es würde viel zu weit führen, wollte ich von den zahlreichen Tierversuchen in PFEIFFers Laboratorium berichten, die diesen menschlichen Experimenten vorausgingen, und von den Hilfshypothesen, die nötig waren, die Wirksamkeit der Substanz und der mannigfaltigen Bedingungen zu erklären. Das Beispiel sollte zeigen, wie es für die Biochemie in Gemeinschaft mit Psychiatrie und Psychologie in langer, rationaler und vorsichtiger Arbeit möglich ist, dem Problem psychischer Wirkungen näher zu kommen, nicht mit spielerischem Ausprobieren im Vertrauen auf einen Zufallstreffer. Was wir zugeben müssen, ist, daß wir zwar einige Bedingungen psychischer Drogenwirkung kennen, aber weit davon entfernt sind, sie in biochemischen oder neurophysiologischen Begriffen erklären zu können.

\section{Methodische Probleme}

a) Tier und Mensch. Für den Rest dieser Vorlesung möchte ich mich mit den methodischen Schwierigkeiten befassen, die sich bei der systematischen Prüfung von Drogen mit psychischer Wirkung bisher ergeben haben.

Zunächst ein paar Worte über die Modellpsychosen, die wir mit chemischen Mitteln erzeugen können. Schwer verständlich ist die Identität der Symptome erzeugt durch chemisch völlig verschiedene Substanzen, wie Meskalin und LSD 25. Einige neuere aktive Psychoto-Mimetica sind Ablömmlinge des Serotonin. - Modellpsychosen sind bisher noch relativ wenig dazu benutzt worden, neue Arzneimittel anf ihre Wirksamkeit beim Menschen auszuprobieren. Der Grund ist wohl, daß es beim Tier nichts mit Modellpsychosen Vergleichbares zu geben scheint. Selbst erhebliche Dosen der beststudierten PsychotoMimetica beeinflussen das tierische Verhalten wenig, auch bei Affen haben Meskalin und LSD 25 keine 
eindeutige Wirkung auf das psychische Verhalten, die sich als dem menschlichen Erleben ähnlich deuten ließe.

Das komplexe Bild der Modellpsychose im Menschen läßt sich mit Phenothiazin-Präparaten unterdrücken; aber auch mit Hilfe naher chemisch verwandter Substanzen, z. B. Brom-LSD, kann man das Auftreten psychischer Symptome nach LSD 25 und nach Meskalin verhindern, wenn man prophylaktisch einige Tage vorher mit mittleren Dosen vorbehandelt (Grnzel und MaYme-Gross 1956). Das mag auf einer Blockierung des Angriffspunktes beruhen, kann aber auch eine Art "Gewöhnung" sein: Es hat sich nämlich gezeigt, daß die regelmäßige tägliche Verabreichung kleiner LSD 25-Dosen, etwa im Laufe einer Woche, auch zur Unterdrückung der psychischen Symptome führen kann (PreIrfere 1958).

Die individuelle Vielfältigkeit menschlicher Reaktionen gegenüber chemischen Mitteln erschwert die Benutzung des Menschen als Versuchstier ganz besonders, wenn es sich um psychische Wirkungen handelt. Jeder Psychiater weiß, wie nötig es ist, z. B. die modernen Antiepileptica am einzelnen Patienten auszuprobieren und auf ihn zuzuschneidern. Hier handelt es sich nur um die Unterdrückung von Anfällen. Wenn es sich um Angstzustände, Verstimmungsepisoden, Reizbarkeit und andere leichtere psychische Anomalien handelt, ist die Variabilität noch erheblich größer.

Jüngst hat ein Verhaltenspsychologe festgestellt, daß seine Ratten schneller und erfolgreicher lernen, sich im Irrgarten zurechtzufinden, wenn er sie regelmäßig täglich streichelt, ehe sie ihren Unterricht haben. Die besten Schüler waren die Ratten, die von der Zeit ihrer Geburt an regelmäBig gestreichelt wurden, weit überlegen ihren GeschwisterRatten, die früh von der Mutter getrennt und nie gestreichelt worden waren (BARRY 1951). Ähnliche Unterschiede sind offensichtlich auch in der Wirkung von Drogen auf die Tiere zu erwarten. Und Massmrmans hat in der Tat zeigen können, weIch erheblichen Einfluß Falktoren der Pflege und sozialen Beziehungen auf die psychologische Arzneiwirkung bei Katzen and Affen haben (vgl. JoNG).

Derartige sekundäre Faktoren können die Beurteilung einer Drogenwirkung auf das Tierverhalten erheblich erschweren, auch wenn die Versuche mit Kontrolltiexen aus demselben Wurf angestellt werden. Was man in Europa den psychologischen Tierversuchen in USA und Rußland vorgeworfen hat, daß sie oft $\mathrm{zu}$ mechanisch und unlebendig im Entwurf und zu stereotyp in der Durchführung seien, ist heute nur bedingt und ausnahmsweise zutreffend. Was die Psychologen und Physiologen aber vergessen, ist die menschliche Herkunft der Worte und Bezeichnungen, die das Verhalten der Tiere beschreiben: Angst, Schuld, Furcht, Aggression, Wut können nur von Menschen verstanden werden, die solche Gefühle introspektiv erlebt haben. Das trifft ebenso zu auf Neid, Ehrgeiz und Eifersucht wie auf Anerkennungsbedürfnis und Neugierde. Begriffe wie Belohnung und Strafe und viele andere haben nur für den Sinn, der die begleitende Stimmungslage kennt. Auch die Verhaltens-Physiologen kommen ohne solche oder ähnliche anthropomorphe Terminologie und Interpretationen nicht aus.

Darüber hinaus aber besteht zwischen Tier und Tier und zwischen Mensch und Tier die sprachlose Verständigung durch den Ausdruck, die man physiognomisch nennen kann, durch die wir mit den Tieren unserer Haus- und Arbeitsgemeinsehaft kommuni- zieren, und solche Kommunikation hört sicher nicht beim Versuchstier auf. ERwIN Straus spricht von ,symbiotischen Verstehen“. „,Die Dürftigkeit unseres beliebig und willkürlich demonstrierbaren und reproduzierbaren Wissens vom Ausdruck steht in einem krassen Gegensatz zu dem Reichtum, der Sicherheit und Abgestuftheit unserer Reaktionen" (E. STRAUS).

b) Menschliche Versuche. Was ich zum methodischen Vorgehen mit menschlichen Versuchspersonen zu sagen habe, mag selbstverständlich klingen und nichts als gesunder Menschenverstand sein. Aber nach Jahren solcher Versuche ist auch die Bestätigung von Bekanntem nicht ohne Interesse.

Zur Frage der Auswahl von Versuchspersonen liegt eine interessante Untersuchung von 29 ,,Freiwilligen“" aus Bethesda vor (Pollns und Peruin 1958). Ein Teil der Versuchspersonen volontierte anstelle der militärischen Dienstpflicht; in 11 Fällen war eine diagnostizierbare psychiatrische Abnormalität nachzuweisen. Wenn dieser hohe Anteil auch durch die besonderen Umstände und Auswahl von WaffenVerweigerern bedingt sein mag, auch unter Studenten ist eine orientierende Exploration durch einen erfahrenen Psychiater vor der Annahme als Versuchsperson notwendig.

Man kann gut verstehen, wenn ein Forscher eine neue Substanz ausprobieren will, daß er verfahren möchte, als ob der Versuch an einer reingezüchteten Tiergeschwisterschaft vollzogen würde. Er sucht nach einer Gruppe von Versuchspersonen mit analogen Kontrollfällen, die sorgsam ausgewählt und zum Vergleich geeignet sind. Beide Gruppen sollen nicht darüber informiert werden, was sie erhalten und was als Wirkung erwartet wird. Überdies sollen die Beobachter, Ärzte und Pflegepersonal, nach Möglichkeit unvoreingenommen und unbefangen sein. Wenn es sich um eine Gruppe von Kranken in der Klinik handelt, sollen die Berichte der Schwestern und Pfleger, die mit den Patienten täglich zusammen leben, sie kennen und beobachten, zuverlässiger sein als das Urteil der Ärzte, die nur einmal am Tage Visite machen und vielleicht wissen oder ahnen, was als Wirkung erwartet wird.

In jüngster Zeit hat ein klinischer Psychologe in England (Foulds 1958) die Ärzte und besonders Psychiater beschuldigt, daß sie vom kontrollierten therapeutischen Experiment viel zu wenig Gebrauch machen. Er zeigt in einer kleinen Statistik, daß die Zahl der positiven Ergebnisse, die zugunsten des Medikaments sprechen, in kontrolliertenVersuchsreihen viel niedriger ist als in unkontrollierten Serien. Dies beweise den Mangel an kritischer Einstellung der Untersucher und die Wertlosigkeit solcher Versuche ohne Kontrolle, nach Ansicht des Verfassers, der ärztlich-ethische Gründe für die Benutzung von Leertabletten als eine Ausrede zurückweist.

Vorwürfe dieser Art haben eine gewisse Berechtigung; aber sie unterschätzen die Schwierigkeiten, Fallen und Schlingen, die jeder zu umgehen hat, der mit Patientengruppen oder auch mit gesunden Versuchspersonen solche Proben anstellt.

Darf ich an die 3 Versuchsanordnungen erinnern, die im kontrollierten Experiment üblich sind: in der ersten werden 2 getrennte, aber vergleichbare Gruppen von Kranken ausgewählt, eine erhält die Arznei, die andere das Placebo, gleichzeitig und mit derselben 
Anweisung. In der zweiten Versuchsanordnung erhalten alle Patienten die Arznei über eine Periode, und nach einiger Zeit, vorher oder nachher, erhalten sie die Leertablette. Die Patienten bilden so ihre eigenen Kontrollfälle; doppelt so viele erhalten das aktive Präparat in der Zeiteinheit - aber Arznei und Kontrolle finden zu verschiedenen Zeiten statt und sind dadurch möglicherweise von Faktoren beeinflußt, die auBerhalb der Arzneiwirkung liegen. Die dritte Versuchsanordnung kombiniert die beiden anderen. Die beiden Gruppen werden wie in der ersten Methode behandelt, bis nach Ablauf einiger Zeit die experimentelle mit der Kontrollgruppe vertauscht wird, jene erhält das Placebo und diese das Präparat.

Methodische Lehrbücher empfehlen die letzte Anordnung als die beste und zuverlässigste.

Wie aus dem vorher erwähnten Artikel hervorgeht und jeder weiß, der medizinische Zeitschriften liest, gibt es aber offenbar eine 4. Methode, über die sich nichts in den Lehrbüchern findet, die aber weitaus am häufigsten im menschlichen Versuch verwendet wird; das Versuchspräparat wird ausgesuchten Patienten gegeben, und der Arzt vergleicht seine Wirkung mit seiner frủheren Erfahrung mit anderen Mitteln in ähnlichen Fällen. Die Vergleichsfälle sind IdealKontrollen aus der Erinnerung des erfahrenen Praktikers, der Zutrauen zu seiner Urteilsfähigkeit hat und dasselbe von seinen Lesern beansprucht. Er: weiß, was er erreichen will and was er von dem Mittel erwarten kann. Er hält sich für kritisch und genügend geschult in der Beobachtung, dab er weder von den Versprechungen des Fabrikanten, noch von der Überzeugungskraft der Anpreisungen irre geführt werden kann. Statistisch mag die Methode anfechtbar sein; aber als ein erster Versuch, die Aktion und Wirksamkeit einer Substanz ausfindig zu machen, Dosis, zeitliche Anordnung und Nebenwirkungen festzustellen, ist sie unentbehrlich.

Das trifft ganz besonders auf Mittel zu, die an poliklinischen Patienten und in der Privatpraxis angewandt werden sollen - in Fällen der kleinen Psychiatrie: pathologische Reaktionen, neurotische Bilder, Persönlichkeits-Abweichungen und psychopathische Entgleisungen. Die Prüfung mit der Idealkontrolle sollte nach meiner Meinung einem jeden systematisch kontrollierten Experiment vorausgehen. Erst wenn das Ergebnis deutlich zugunsten des Mittels ausfällt, ist die systematische Prüfung gerechtfertigt. Fällt es negativ aus, so wird es vergessen und leider oft nicht veröffentlicht. Das ist wohl der Grund, weil unser kritischer Psychologe so wenige Artikel mit negativem Resultat für seine Statistik fand.

Jeder, der versucht hat, eine wohlkontrollierte Prüfung eines Mittels in der Praxis oder Poliklinik anzustellen, stößt auf die erheblichen Schwierigkeiten der Unsicherheit der Placebowirkung und des Einflusses von Umweltfaktoren, denen der ambulante Patient ausgesetzt ist, und besonders auf die mangelnde $Z u$ verlässigkeit der Selbstbeobachtung des Patienten. Dazu einige Bemerkungen:

Die Wirkung von Leertabletten war in letzter Zeit dass Thema vieler Debatten und einiger Publikationen; die Verfasser glauben, man könne diese Wirkung standardisieren, und einer meiner Mitarbeiter schlug vor, die Placebo-Wirkung an ein paar Hundert psychiatrisch Kranker verschiedenster Art ein für allemal klarzustellen und festzulegen. Jede Arznei, die diese Wirkung übertreffe, verdiene weitere Prüfung.

Dieser Vorschlag mag statistisch einleuchten, aber er übersieht völlig, daß die Wirkung nur zum kleinsten Teil auf die Tablette zurückgeht, sondern auf die Worte, den Tonfall und Ausdruck des Doktors, die Ausstattung seines Sprechzimmers, die Selbstsicherheit der Empfangsdame, die Miene der Krankenschwester und auf alle Umstände und Nebenumstände der Verabreichung. Alles dieses müßte standardisiert werden!

Jüngst erregte der Bericht über eine Drogenprüfung in London die öffentliche Aufmerksamkeit, weil sie angeblich die Wirkungslosigkeit einiger der neuen "Tranquillizers" demonstrierte. Das Experiment war von einem der besten biologischen Statistiker in England entworfen und überwacht. Die Frage der Leertabletten wurde dadurch umgangen, daß man einige der neuen Medikamente mit einem Barbiturat (Luminal) verglich, außerdem auch mit dem Placebo. Die verschiedenen Mittel wurden in der statistisch vorbereitenden Folge miteinander abgewechselt - auch die Arzte wuBten nicht, was der Kranke in jeder Woche erhielt, sie notierten ihre Beobachtungen regelmäßig, die sich allein auf die subjektiven Eindrücke des Patienten stützten. Wie man hätte voraussagen können, die Kranken fühlten sich weitaus am besten mit Luminal, das eine gute Stimmung hervorruft; nach einem Glas Whisky hätten sie sich noch besser gefühlt, wenn der Zweck der Behandlung nur die Erzeugung von Euphorie gewesen wäre. Über den Wert der anderen Drogen sagte der Versuch nichts aus.

Einwandfreie und befriedigend kontrollierte Prüfungen lassen sich nur an hospitalisierten Patienten anstellen, und die großen Nervenanstalten, in denen Kranke über Jahre unter gleichförmigen Bedingungen und ständiger Beobachtung leben, scheinen ideal für solche Versuche. Zwei meiner Mitarbeiter haben jüngst in einer kleinen Anstalt eine Gruppe von etwa 30 chronischen Patienten ausgewählt, die seit über 5 Jahren keinerlei Behandlung Eörperlicher Art und keinerlei Pharmaka erhalten hatten; die Untersucher formten 2 Vergleichsgruppen nach Alter, Krankheitsart und -dauer und Schwere des Zustandes, sorgfältig aufeinander abgestimmt. Während des Versuches zeigte sich in unerwarteter Weise, daB der Blutdruch und das Körpergewicht durch die Droge beeinflußt wurden. Die sonst so wohl aufeinander abgepaßten Vergleichspaare erwiesen sich als fundamental verschieden in bezug auf diese beiden Faktoren und als signifikant unvergleichbar im einzelnen Paar wie im Durchschnitt.

Bei einer früheren Gelegenheit (1957) habe ich beschrieben, wie das Pflegepersonal in einer der vielfach gebrauchten amerikanischen Verhaltens-Skalen unterrichtet wird. Die Skala registriert etwa 10 Verhaltungsweisen des Patienten, sein Verhalten zur Arbeit, zur Behandlung, zum Mitpatienten, Pflegepersonal, bei der Nahrungsaufnahme usw. Für jedes Verhalten sind 5 Grade gegeben, die Pflegerin hat das Zutreffende anzuhaken. So ergibt sich in relativ kurzer Zeit ein dokumentiertes Beobachtungsmaterial, das sich statistisch auswerten läßt. Aber wie immer bei der Anwendung von Skalen und Fragebogen, sie 
lassen keinen Spielraum für die ungewöhnlichen Vorkommnisse, sie schärfen nicht das Auge des Beobachters, sondern machen ihn eher gleichgültig und versehen ihn mit Scheuklappen.

Das Folgende illustriert eine Zuverlässigkeitsprobe von Pflegeberichten: Meine Mitarbeiter wollten ihren chronischen Patienten etwas geben, das einen deutlich erkennbaren. physischen Effekt hatte, ohne das seelische Verhalten zu beeinflussen. Sie wählten anstelle des Placebo eine kleine Gabe von Nicotinsäure (GREGORY 1957), das in einer Menge von $100 \mathrm{mg}$ vorübergehendes Erröten hervorruft, sonst keine Wirkung auf die Erkrankung hat. Das Pflegepersonal hatte die Anweisung, auf gewisse Zeichen psychischer Änderung zu. achten, darunter auch auf Erröten.

Positive Berichte über beobachtetes Erröten kamen nur langsam; nur 2 Patienten von den 14, die Nicotinsäure erhielten, waren in der 1 . Woche gemeldet, mehr in der folgenden. usw., bis schließlich in der 7 . Woche 10 von 14 korrekt beob. achtet waren. Aber im Laufe der 3. und folgenden Wochen tauchten auch falsche positive Meldungen von Patienten auf, die keine Nicotinsäure erhielten und als errötend gemeldet wurden.

Solche Fehlreporte erschienen später und wurden länger aufrechterhalten. Trotz dieser mehr oder weniger korrekten Berichte über das Erröten hatten diese Beobachtungen wenig Einfluß auf die Gesamtbeurteilung der Arzneimittelwirkung durch das Personal - die Pfleger waren fähig, die beiden Beobachtungen getrennt zu halten. -

Diese Mitteilungen aus der Werkstatt der Psychopharmakologie sollen zeigen, wie wir uns, wie viele andere, bemühen, eine kritische Methode zu entwickeln, trotz Schwierigkeiten und theoretischer Bedenken. Nicht nur Pfleger und Schwestern, Mitpatienten, Angehörige, die oft regelmäßig besuchen, und schließlich der Kranke selbst sollte über die Wirkung der Medikation gehört werden. TonbandAufnahmen dieser Aussagen sind besonders wertvoll, weil man sie in Ruhe auswerten und vergleichen kann, sie haben sich in unseren Versuchen besonders bewährt, um stereotype, angelernte Phrasen der Pflegerberichte los zu werden. Nichts ist im Augenblick zu banal, und nur durch große Sorgfalt im einzelnen ist Fortschritt möglich.

c) Der Versuchsleiter. Das wichtigste aber in allen menschlichen Experimenten ist und bleibt die Ausbildung von Ärzten und Wissenschaftlern in der Fähigkeit kritischer und unterscheidender Beobachtung, die zwischen Selbstvertrauen und Selbstkritik die richtige Mitte hält. Gesundes klares Urteil darf nicht durch allzu reichlich gegenwärtiges Erfahrungswissen beeinflußt sein, Naivität und Offenheit für alles Neue muß durch Kenntnis und Einsicht kontrolliert werden. Das geschulte Urteil des kritischen Beobachters ist unersetzbar in allen. Gebieten der Forschung, aber besonders in der pharmakologischen Psychiatrie aber es kann und sollte geschult werden. Es ist nicht zu ersetzen durch blinde oder doppelt blinde Experimente, durch Massenbeobachtung und ihre statistische Auswertung, durch mechanische und vereinfachende Prozeduren, die die komplexe Variabilität des Versuchstieres nicht berücksichtigten. „Einzelbeobachtung und Massenversuche schließen sich nicht aus, sondern ergänzen sich in der Psychologie und Psychiatrie."

Schließlich müssen wir uns gegen den modischen Pessimismus zeitgenössischer Philosophie wehren, der sich auch auf die Anwendung der Pharmakologie in der Psychiatrie ausbreitet. Davon gibt es anscheinend 2 Spielarten, Die eine arbeitet in kurzen hektischen Stößen: Eine Hypothese wird ergriffen und mit
Enthusiasmus auf das ganze Erfahrungsfeld ausgedehnt - alle Patienten werden mit dem gleichen vielversprechenden Mittel behandelt, alles andere, früher erprobte verlassen und anscheinend vergessen. Wenn die Ergebnisse den Erwartungen nicht entsprechen, was sie bei der indikations- und kritiklosen Anwendung schwerlich können, schlägt die ganze Einstellung um - mit dem Badewasser wird auch das Baby ausgeschüttet. Man ist für eine neue Theorie bereit - von der alten bleibt kaum etwas lebendig.

Die 2. Spielart des modischen Pessimismus ist gescheiter und gefährlicher. Die erheblichen methodischen Schwierigkeiten, so sagen uns diese Philosophen, beruhen auf einem grundsätzlich falschen theoretischen Ansatz: Seelische Vorgänge sind nicht mit naturwissenschaftlichen Mitteln anzugreifen. So wie KrafePeLIN am Ende des vorigen Jahrhunderts wenig Fruchtbares mit seinen Giftversuchen zutage brachte, so wird die gegenwärtige pharmakologische Richtung eine Episode bleiben, es sei denn, daß sie ihre Phänomene auf eine philosophisch-anthropologische Weise interpretiert und sich von den Naturwissenschaften trennt. Diese neo-romantische ExistentialAnalytik hat in der deutschen Psychiatrie die Einbildungskraft mancher jüngerer Forscher entzündet. Wenn man an die geschilderte mythische Vergangenheit psychomimetischer Arzneien denkt, muß man auf eine literarische Flut anthropologischer Ausdeutungen vorbereitet sein. Die Psychoanalyse hat sich bereits des LSD 25 bemächtigt und seine Enthüllungen aus dem Unbewußten als Heilmittel begrüßt. Ähnliche, wenn auch viel kompliziertere Interpretationen der Modellpsychose und anderer Wirkungen psychischer Pharmaka kann man von den Schülern des neoromantischen Obskurantismus erwarten.

Eine der großen Schwierigkeiten auf unserem Gebiet liegt ohne Zweifel in der Doppelstellung als Wissenschaftler und Arzt, die dem Psychiater zugemutet wird, wenn er psychopharmakologisch arbeitet. Der Kranke verlangt jetzt und hier Hilfe, der Fabrikant und die Industrie machen mit mehr oder weniger Berechtigung von diesem Verlangen Gebrauch. Der wissenschaftlich geschulte Arzt wartet auf den Beweis der Wirksamkeit und Unschädlichkeit, ehe er das Mittel verschreibt, nach dem der Patient verlangt. Bis hierhin unterscheidet sich die Situation nicht von ähnlichen in der allgemeinen Medizin, wo die Lösung heutzutage wenig Schwierigkeit bereitet. Die Wirkung von Faktoren wie Hoffnung, Erwartung, Glauben, Vertrauen, Überzeugungskraft und ähnlicher seelischer Regungen auf das Befinden und die Symptome des psychiatrisch Kranken verschleiern und verwischen die Zusammenhänge und tragen $\mathrm{zu}$ der Unsicherheit des wissenschaftlich eingestellten Arztes bei. Um so mehr brauchen wir eine verbesserte und klare Methodik und die Kombination von Nüchternheit und Einbildungskraft, die in der Körpermedizin zur Úberwindung der Doppelstellung geführt hat.

Literatur. Barny, H.: J. comp. physiol. Psychol. 50, 366 (1957). - Beringer, K. : Der Meskalinrausch. Berlin 1927. Studium gen. 1, 281 (1948). - BLEULER, M. : Neuro-pharmakologische Hypothesen in ihrer Bedeutung für die Schizophrenielehre, Collegium Internationale Neuropsychopharmacologicum, Rome 1958. - Drabkin, D. L.: Thudichum, Chemist of the Brain. Philadelphia: University Philadelphia Press 1958. - Foulds, G. A.: J. ment. Sci. 104, 259 (1958). - 
GrNzeL, K. H., and W. Maxer-Gross: Nature (Lond.) 178, 210 (1956). - GREcory, I.: J. ment. Sci. 101, 85 (1955). Hofter, A., H. Osmond and J. Smythies: J. ment. Sci. 109, 29 (1954). - JUNG, R.: Klin. Wschr. 36, 1154 (1957). Kratepilin, E.: Die Beeinflussung einfacher psychischer Vorgänge durch einige Arzneimittel. Leipzig 1892. - LETEMENDIA, F., T. HARRIS and W.MAYER-GROSS: Comparison of abnormal behavioral states induced by drugs in animals and men, Collegium Internationale Neuropsycho-pharmacologicum, Rome
1958. - Mayer, Gross, W.: Nervenarzt 28,97 (1957). Murphy, H. B., E. H. J maney and Carr Pfeifher: 2-Dimethylaminoethanol as a central nervous stimulant - one aspect of the pharmacology of reserpine. Meeting of Assoc. Res. Nerv. and Ment. Dis. New York, December 1957. - PFerffer, Carl: Persönliche Mitteilung 1958. - Poldiv, W., and S Pertin: Amer. J. Psychiat. 115, 129 (1958). - Stolx. W. A.: Schweiz Arch. Neurol. Psychiat. 60, 279 (1947). Strads, E.: Vom Sinn der Sinne. Berlin 1935.

\title{
DURCH ARTHROPODEN ÜBERTRAGENE VIRUSER KRANKUNGEN DES MENSCHEN
}

Von

\section{Max Themer und Tordi Casals}

\author{
Rockefeller Foundation Virus Laboratories, New York
}

Das Gelbfieber ist die erste Infektionskrankheit des Menschen, von der gezeigt werden konnte, daß sie auf ein Virus zurückzuführen ist und durch Insekten übertragen wird. Diese grundlegenden Beobachtungen wurden um die Jahrhundertwende von WALTER REED u. Mitarb. ${ }^{1}$ gemacht. Sie zeigten unter Verwendung freiwilliger Versuchspersonen, daß der gewöhnliche tropische Hausmoskito Aedes aegypti das Gelbfieber überträgt. Der Moskito kann vom Menschen nur während der ersten Tage der Frkrankung infiziert werden und den Erreger erst nach einer Zeit von ungefähr 12 Tagen weitergeben. Da für diese Untersuchungen menschliche Versuchspersonen verwendet werden mußten, war die Zahl der Experimente beschränkt.

Doerr, Franz u. Taussta ${ }^{2}$ zeigten 1909, daß das Phlebotomusfieber der Adriaküste durch den gewöhnlichen Phlebo. tomus papatasii übertragen wird. Auch für diese Versuche muBten wegen des Fehlens geeigneter Versuchstiere freiwillige Versuchspersonen verwendet werden. Das Dengue-Fieber war die dritte Viruskrankheit, bei der die Übertragung durch Insekten nachgewiesen werden konnte. In sorgfältig durchgeführten Untersuchungen an männlichen Freiwilligen konnten Srler, Hali u. Hrichens ${ }^{3}$ (1926) zeigen, daß das DengueFieber auf den Philippinen durch Aedes aegypti übertragen wird. Die Bedingungen, unter denen ein Moskito die Infektion mit Dengue übertragen kann, waren ähnlich denen beim Gelbfieber, denn der Moskito kann die Infektion nur während der ersten Tage der Erkrankung vom Menschen aufnehmen und sie erst nach einem Zeitraum von ungefähr 12 Tagen weitergeben.

Die Entdeckung, daß der Rhesusaffe (Stokes, Batkr $u$. HuDson ${ }^{4}$ ) und die weiBe Maus (THEILER ${ }^{5}$ ) empfindlich für das Gelbfiebervirus sind, eröfnete eine neue Ära für die Untersuchung der durch Insekten übertragenen Viruserkrankungen. In schneller Folge wurde eine Zahl von Viren von Menschen und Tieren isoliert und als Ursache von Erkrankungen erkannt. Hierher gehören die St. Louis-Encephalitis $^{6}, 7$, die japanische B-Encephalitis ${ }^{8-10}$, die westliche Pferdeencephalomyelitis ${ }^{11}$, die östliche Pferdeencephalomyelitis ${ }^{12}, 13$, die russische Frühling-Sommer-Encephalitis ${ }^{14}$ und die Louping ill $^{15}$. Alle diese Erreger wurden aus dem Zentralnervensystem tödlich erkrankter Menschen und Tiere gewonnen; später wurde der Beweis geliefert, daß diese Viren durch den Biß von Insekten übertragen werden. Während der Untersuchungen der Epidemiologie des Gelbfiebers in Uganda wurden 2 Erreger aus dem Blut von Menschen isoliert, die an einem Fieber unbekannter Ursache litten. Das eine von ihnen, später Bwamba-Fieber-Virus genannt, wurde mehrfach isoliert und als Ursache dieser Erkrankung festgestellt ${ }^{\mathbf{1 6}}$. Das andere, das westliche Nil-Virus ${ }^{17}$, wurde nur einmal gewonnen. Durch serologische Untersuchung wurde bewiesen, daß es sich hier um den Erreger dieser Erkrankung handelt. Von beiden wurde später gezeigt, daß sie durch Moskitos übertragen werden.

Außer diesen von infizierten Wirbeltieren gewonnenen Erregern wurden mehrere Virusarten aus wild gefangenen Moskitos isoliert. Diese Erreger wurden zufällig entdeckt während epidemiologischer Studien über Gelbfieber und St.-LouisEncephalitis. Derartige in Afrika entdeckte Brreger waren Bunyamwera ${ }^{18}$, Uganda- $\mathrm{S}^{19}$, Ntaya ${ }^{20}$, Semliki-Forest ${ }^{21}$ und Zika. In Südamerika wurden 4 Erreger isoliert, die die Namen Anopheles A und B, Wyeomia ${ }^{23}$ und Ilheus ${ }^{24}$ erhielten. In Nordamerika wurde das kalifornische Encephalitis-Virus von Hammon u. Rerves ${ }^{25}$ entdeokt. Serologische Untersuchungen haben gezeigt, daß Antikörper gegen alle diese Erreger (mit 3 Ausnahmen) im Blut von Menschen gefunden werden, die in der Gegend leben, wo die Viren isoliert wurden. Hierdurch wurde mit aller Wahrscheinlichkeit gezeigt, daB diese Erreger die Ursache menschlicher Infektionen sind. Die 3 Ausnahmen waren Anopheles A, Anopheles B und Wyeomia. Über diese 3 Erreger sind bisher jedoch wenig Untersuchungen angestellt worden.

SMrthburn zeigte als erster eine gewisse immunologische Überlappung zwischen den durch Insekten übertragenen Viruserkrankungen. Durch Neutralisationsteste konnte er Antigenverwandtschaften zwischen dem japanischen B-, dem St. Louisund Westnilvirus zeigen ${ }^{26}$. CaSALS bestätigte dies durch Komplementbindungstests ${ }^{27}$. Außerdem zeigte er, daß die Viren der russischen Frühling-Sommer-Encephalitis und des Louping ill nahe verwandt sind. Havens u. Mitarb. 28 zeigten Kreuzreaktionen zwischen den Erregern der östlichen Pferdeencephalomyelitis und der westlichen Pferdeencephalomyelitis. Mittels des Komplementbindungstestes zeigten HUGHES $u$. Mitarb. ${ }^{29}$, daß das Tlheus und $\mathrm{St}$. Louis-Virus verwandt sind und SABIN zeigte Beziehungen zwischen den japanischen B., West-Nil-, Dengue- und Gelbfieberviren ${ }^{30}$. Diese Beobach" tungen zeigten deutlich, daß die durch Arthropoden übertra. genen Viren nicht vollkommen verschiedene Einheiten sind, sondern dab sie immunologisch verwandt und daher wahrscheinlich auf gemeinsame Entwicklungsstufen zurückzuführen sind.

Die große Zahl der Variationen der durch Insekten übertragenen Viren, die breite geographische Verteilung und die immunologische Verwandtschaft zwischen einigen von ihnen bestimmten die Rockefeller-Foundation, eine Untersuchung der durch Insekten übertragenen Viruserkrankungen des Menschen und seiner Haustiere in der ganzen Welt zu veranstalten. Zu diesem Zweek wurde die Arbeit der Viruslaboratorien der Rockefeller-Foundation in New York mit Außenstationen in Südamerika, Afrika und Indien koordiniert. Gegenwärtig arbeiten 5 derartige Laboratorien. Zwei davon befinden sich in Südamerika, eines in Port of Spain, Trinidad, in Zusammenarbeit mit dem Health-Department der Regierung von Trinidad und Tobago und dem Colonial Research and Development Scheme; das andere in Belem in Brasilien, in Zusammenarbeit mit dem Servico Especiales de Saude Publica. In der Südafrikanischen Union wird in Johannesburg eine weitere Station gemeinsam mit dem South African Institute for Medical Research unterhalten. In Indien arbeitet eine Station in Poona eng mit dem Indian Medical Research council zusam. men. Eine weitere befindet sich in Berkeley, Kalifornien, in Verbindung mit dem State Department of Health. Seit dem vor mehreren Jahren einsetzenden Beginn dieser Untersuchungen wurde eine große Zahl von durch Insekten übertragene Viren isoliert und gegenwärtig weiter untersucht. Es ist die Aufgabe dieser Utbersicht, den gegenwärtigen Stand unseres Wissens uber diese Viren darzustellen. Viele der hier angeführten Untersuchungen wurden durch die RockefellerFoundation durchgeführt; hoffentlich sind bedeutende Beobachtungen von anderer Seite hierbei nicht übersehen worden.

\section{Einteilung}

Durch Insekten übertragene tierische Virusinfektionen umfassen eine Gruppe von Viren, die in der Natur zur Vermehrung im Körper sowohl von Vertebraten - Säugetieren und Vögeln - als auch von Nichtwirbeltieren - Arthropoden fähig sind. Jedoch zeigen nur die Vertebraten eine Erkrankung oder andere Zeichen einer Infektion, während die Insekten keine erkennbare Schädigung oder Erkrankung aufweisen. Das Insekt ist lediglich der UUberträger und wird durch die Nahrungsaufnahme infiziert zu einer Zeit, wenn 